\title{
MEMS AND MORE FOR THE BRAIN: THE CLUSTER OF EXCELLENCE BRAINLINKS-BRAINTOOLS AT THE UNIVERSITY OF FREIBURG
}

\author{
O. Paul* and P. Ruther \\ Microsystem Materials Laboratory, Department of Microsystems Engineering (IMTEK), \\ University of Freiburg, Germany
}

\begin{abstract}
In the fierce competition among German academic institutions in the national Excellence Initiative, the University of Freiburg has successfully defended the excellence cluster project BRAINLINKS-BRAINTOOLS. The cluster benefits of 28 Mio $€$ of funding for the period from Nov. 2012 to Oct. 2017. Over 40 principal investigators of the University Clinic Freiburg and the Faculties of Biology and Engineering are teaming up, involving about a hundred young scientists. The cluster aims to bring the state of the art in bi-directional brain-machine interfaces to a new level. Its research program merges results on the neurocomputational foundations, novel MEMS tools, and computer science methods into platforms designed for clinically relevant applications. With Parkinson's disease, epilepsy, stroke, and paralysis, four major neurological disabilities are addressed.
\end{abstract}

\section{INTRODUCTION}

Whether the brain is the last frontier of science, as has been stated, may be questioned. However, without any doubt, its about $10^{11}$ neurons and their $10^{14}$ interconnections constitute a multiscale system of awe-inspiring complexity leaving much to discover.

Large project frameworks, such as the Human Brain Project (HBP) in Europe or the Brain Initiative in the USA have outlined steps to tackle this formidable challenge. HBP has been originally designed to trigger research investments at a level of about one euro-cent per neuron, while the Brain Initiative starts with an initial expenditure of 100 Mio US\$ in 2014 and is planned for a duration of ten years.

Other leading centers of neurotechnology pursue more focused endeavors. Among them are the NSF Engineering Research Center for Sensorimotor Neural Engineering (CSNE) at the University of Washington, Seattle, emphasizing the development of neural control systems for prosthetic limbs; the Edmond and Lily Safra Center for Brain Sciences (ELSC) in Jerusalem, Israel, covering all aspects of neuroscience including computational neuroscience as well as motor BMI and DBS intervention; and thirdly, the Australian Bionic Vision Consortium (BVC) with its focus on visual prosthetics.

\section{THE BRAINLINKS-BRAINTOOLS CLUSTER Aims}

The overall scientific goal of BrainLinks-BrainTools [1] is to to raise the bi-directional interaction between technical instruments and the brain to a new level by developing flexible yet stable, and adaptive yet robust hybrid brain-machine interfaces. With funds amounting to $28 \mathrm{M} €$ for five years, the cluster builds on the existing competence at the University of Freiburg and the University Clinic Freiburg in

- Neuroscience (Bernstein Center Freiburg; Department of Biology),

- MEMS engineering (Dept. of Microsystems Engineering [IMTEK], Faculty of Engineering),

- Artificial intelligence, machine learning, and robotics (Dept. of Computer Science [IIF], Faculty of Engineering),

- Clinical neuromedicine (University Clinic Freiburg), and

- Ethics of science and technology (Institute of Ethics and
History of Medicine, Faculty of Medicine). It combines the corresponding activities into a tightly knit research program. As a full-range university, the University of Freiburg thereby materializes its mission statement to contribute solutions to the pressing interdisciplinary challenges of our time. With BRAINLINKS-BRAINTOOLS it does so by establishing a strong link between engineering, the life sciences, and philosophy.

The cluster's scientific and technological activities are centered on clinical scenarios addressing four major neurological pathologies, namely Parkinson's disease, epilepsy, stroke, and paralysis. The approach to these disabilities is guided by two long-term visions for intelligent neuroprosthetic technology platforms. These are:

- Prosthetic limbs with Neural Control (LiNCs) defining brain-controlled assistive devices and prostheses to return movement capacity in paralyzed patients and amputees, and to improve the rehabilitation of patients suffering from stroke or brain trauma;

- Smart Energy-Autonomous Micronodes (SEAMs) targetting implantable, autonomous devices with closed-loop feedback through implanted recording and stimulation for the treatment of neurological disorders originating in pathological network structures and activity dynamics, such as epilepsy and Parkinson's disease, among others.

These two platforms will rely on far more than just novel MEMS devices. They will capitalize heavily on methods of computer science, especially in view of LiNCs with minimally invasive brain-machine interfaces, and of data evaluation and action planning needed for SEAMs.

\section{Structure}

The cluster is structured into three research areas and a central service and management unit. It relies on a relatively lean management and invests the dominant part of its funds directly into research and into strategic support measures ultimately benefiting research as well.

Research Area A 'Foundations' addresses the neurocomputational foundations, both in theory and experiment. The aim is to identify optimal strategies as to when, where, and how to interact with the brain, whether it is to read out information or induce intended changes in the activity of neural networks, from local circuit units to large-scale networks spreading across multiple brain areas. A range of techniques, from network modeling to functional magnetic resonance imaging (MRI), electrophysiology, and optogenetic tools, applied to in vitro and in vivo models, are used.

Research Area B 'Core Technologies' focuses on the development of multifunctional technical tools for interfacing with the brain, based on methods of microsystems engineering and computer science. Tailored for spatial resolutions from $30 \mu \mathrm{m}$ to $5 \mathrm{~mm}$, the tools are designed to be deployed in various brain regions, from the cortical surface to deep brain structures, both in view of monitoring neuronal activity and modulating, i.e., stimulating or inhibiting, it. The electrical, magnetic, and optical signal domains are being addressed. Advanced algorithms for signal processing and interpretation, data handling, and action planning turn the tools into stable, adaptive and robust neurotechnological interfaces. 
Thirdly Research Area C 'Applications' pursues the objective of developing therapies and paradigms transferring the results of Research Areas A and B into novel preclinical and clinical applications in the context of the above-mentioned neurological pathologies.

\section{Strategic measures}

BRAINLINKS-BRAINTOOLS has formed a strategic axis with the CSNE, the ELSC, and the BVC.

In addition, the cluster has already four out of five young research leader positions with candidates pursuing a research program of their design and endowed with funds for building up a small research teams for the duration of the cluster.

Three new professor positions have been defined and are currently being filled in the areas of

- Optophysiology (Faculty of Biology),

- Functional models and multi-scale networks (Faculty of Medicine), and

- Neurorobotics (Faculty of Engineering), in order to round off the University of Freiburg's academic portfolio in neurotechnology and neuroscience.

Furthermore, the cluster has nationally and internationally teamed up with a large group of academic, clinical, and industrial application centers for training, dissemination, and transfer. It is open to further collaborations.

\section{MEMS DEVELOPMENTS}

Projects with durations of one year ('exploratory projects') and up to three years ('advanced projects') are funded based on periodic calls for proposal with a review-based selection process. In the following we outline currently funded projects. Further information is available online [1].

\section{Current MEMS projects in BRAINLINKS-BRAINTOOLS}

Project AdvancedEDC pushes the state of the art in highly integrated, electronically switchable, intracortical probe arrays for recording and stimulation. Advances over current electronic depth control (EDC) probes [2] aim at increasing (i) the reconfiguration flexibility and speed for switching between electrode configurations, (ii) the spatial resolution by using commercial $0.18 \mu \mathrm{m}$ CMOS technology, (iii) the signal quality by the on-shaft cointegration of preamplifiers, filters, and analog-to-digital converters (ADC), and (iv) the number of simultaneously extracted neural signals up to 32 at least. Probes are tailored with respect to functionality and shaft dimensions to the needs of the neuroscientific experiments pursued by the cluster partners. Studies of mouse epilepsy for instance impose particularly severe restrictions on the probe dimensions.

The probe hardware will be complemented by dedicated insitu microelectronic hardware for executing neurocomputational tasks, and by algorithms for the analysis and classification of neuronal signals, for data reduction and feedback policy learning.

Project COLUMNS addresses the development of noninvasive light sources for optogenetic experiments. Light beams shaped as Bessel beams [3] offer the property of beam selfreconstruction when penetrating diffractive media. Beam shaping is achieved by conical axicon elements [3] with thermally controlled focal lengths. Laser diodes will serve as light sources. A dense array of Bessel beams will allow one to generate the spatiotemporal patterns within the brain tissue required for a wide variety of optogenetic studies.

Similarly, project WOLDOR aims at the development of intracortical and epicortical optrodes, i.e., light sources for optogenetic experiments combined with recording electrodes. The ap- proach is different, however, since the intracortical probes will be of the penetrating type, based on slender probe shafts [4], while the epicortical structures will capitalize on established fabrication techniques for PDMS rubber and polyimide (PI) based flexible epicortical grid (ECoG) arrays [5]. The project focuses on the development of fabrication, packaging, and testing techniques. Light sources with optogenetically relevant wavelengths, e.g., $450 \mathrm{~nm}$, will be integrated into these multifunctional structures. The systems are electrically controlled and thus avoid the clumsy optical links via glass fibers of conventional optogenetic probes.

Project MAGRITE focuses on MEMS based micro-coils and culture chambers for in-situ high-resolution magnetic resonance imaging (MRI) studies of epileptogenesis in mouse brain slices. The studies aim at elucidating structural and molecular changes associated with the development of epileptic seizures. Micro-coil fabrication relies on wire-bonding and polymer micromachining [6]. Resolutions down into the $10 \mu \mathrm{m}$ range have so far been achieved and further improvements by design optimization and implementation of novel spin hyperpolarization techniques [7] can be expected.

Compatibility with functional MRI (fMRI) is a definite advantage, if not a necessity, for any neural probe components. To support the development of fMRI compatible tools, project NEUMARE builds a database of MRI relevant properties of materials used for probe fabrication and identifies rules for judicious probe design. The materials include $\mathrm{Al}_{2} \mathrm{O}_{3}$, diamond-like carbon $\mathrm{SiC}, \mathrm{Si}, \mathrm{IrO}_{\mathrm{x}}, \mathrm{Fe}, \mathrm{Cu}, \mathrm{Au}$, solder alloys, Mn, Mo, Pt, W, PDMS, PI, and teflon, among others [8]. In composite structures, magnetic susceptibility jumps between these materials in the ppm range can cause image distortions and artifacts, which require minimization by simulation and design optimization.

Project SEAM-TEG addresses the issue of in-situ energy scavenging for powering implanted neurotechnical components. It attempts to reach this goal by thermoelectric energy conversion using the temperature gradient between the body and the ambient to which subcutaneous tools may be exposed. Thermopiles based on electroplated $\mathrm{Bi}_{2} \mathrm{Te}_{3}$ and $\mathrm{Sb}_{2} \mathrm{Te}_{3}$ [9] are explored as a promising choice with acceptable energy conversion efficiencies.

New projects NeuroTRACEIT, MAKE IT ReaL, and SEAM$W i T$ propose to concentrate, respectively, on: the controlled release of fluorescent dies from polymer (PEDOT) electrodes for the precise post-mortem localization of neurons from which recordings were previously made; next-generation micro-ECoGs for the closed-loop recording and stimulation in an awake sheep model as a paradigm for the chronic functional testing of neural implants; the development of a powerful, versatile telemetry unit for energy and bi-directional data transfer with implantable neural probes.

\section{Neurotechnology by the Microsystem Materials Laboratory Probe fabrication approach}

Silicon based neural probes for intracerebral recording have established themselves as valuable and reliable tool for acute and sub-chronic neuroscientific experiments. Probes by the pioneering groups at the University of Michigan [10] and University of Utah [11] have been used by many researchers and are being commercialized by NeuroNexus and Blackrock Microsystems, respectively. Fabrication of the Michigan probes has traditionally made use of heavily B-doped silicon shafts with thicknesses around $15 \mu \mathrm{m}$ fixed on a thicker base and connected to the external instrumentation by ribbon cables based on the same highly doped material. In the case of the Utah probes the fabrication has proceeded by ion migration through thicker silicon substrates than standard wafers followed by trenching using dicing, and sharpening by isotropic etching [11]. 
A third approach benefiting of similar design degrees of freedom in view of the lateral shaft dimensions as the University of Michigan's, but at the same time offering design freedom regarding shaft thickness as well, has been proposed by the authors' team [12]. It is termed etch-before-grinding (EBG). After completion of the thin film deposition and structuring processes necessary for the definition of the recording sites and interconnection leads as well as their passivation, the probe shape is defined by deep reactive ion etching (DRIE) of the wafer front; the intended probe thickness is obtained by rear wafer grinding. Electrode metalizations apply either $\mathrm{Pt}$ or $\mathrm{IrO}_{\mathrm{x}}$ combined with $\mathrm{Ti} / \mathrm{Au} / \mathrm{Pt}$ layer sandwiches used for interconnection leads along the slender probe shafts. Probes with shaft lengths between 1 and $40 \mathrm{~mm}$, widths down to $30 \mu \mathrm{m}$, and thicknesses down to $25 \mu \mathrm{m}$ have been demonstrated. Impedances for electrodes sites with areas of $962 \mu \mathrm{m}^{2}$ at $1 \mathrm{kHz}$ are typically $1.2 \mathrm{M} \Omega$ and $140 \mathrm{k} \Omega$ for $\mathrm{Pt}$ and $\mathrm{IrO}_{\mathrm{x}}$ electrodes, respectively [13][2]. Examples of probes are shown in Fig. 1.

Similarly fabricated probes with integrated fluidic channels [14] and biosensors [15] have been realized by dual-side DRIE of Si combined with additional Si micromachining, bonding processes, and integration of dedicated sensing layers.

\section{Electronic depth control probes}

Using the above techniques it is straightforward to realize probes with integrated microelectronic circuitry as well. To this end, before the EBG process, the substrates are processed using commercial CMOS technology. The advantage is that a vastly larger number of electrode sites can be integrated along the shafts, each site switchable to a given number of output lines. In shafts with $4 \mathrm{~mm}$ lengths realized in a $0.6 \mu \mathrm{m}$ CMOS technology by XFAB, Erfurt, Germany, 188 sites can each be switched to two among eight output lines. The switches are standard transmission gates controlled by D flip-flops linked as a shift register [2]. The switch arrangement allows any configuration of two tetrodes and a large number of configurations with eight sites scattered along the shaft to be selected. Combs with four such shafts are available as well. Since the microelectronic components are distributed along the shafts, the probe systems benefit of a relatively small base, which facilitates probe usage in sub-chronic experiments [16]. EDC probes are shown in Fig. 1(b).

In AdvancedEDC this state of the art is being pushed further by the use of 6 -metal $0.18 \mu \mathrm{m}$ technology by X-FAB. This technology node will enable significantly more smartness to be integrated into the probe shafts and bases.

\section{Optrodes}

Optogenetics has emerged as an extremely fruitful technique enabling experiments where neuronal activity is triggered or inhibited by optical signals rather than electrochemically [17]. This is achieved by viral transfection of neurons with optically sensitive (a)

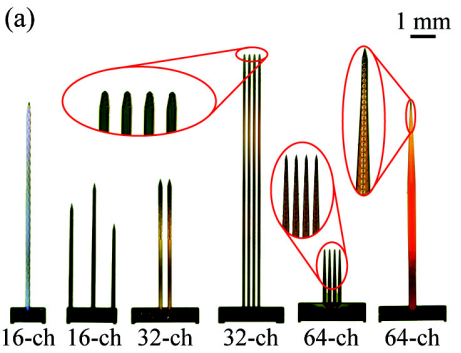

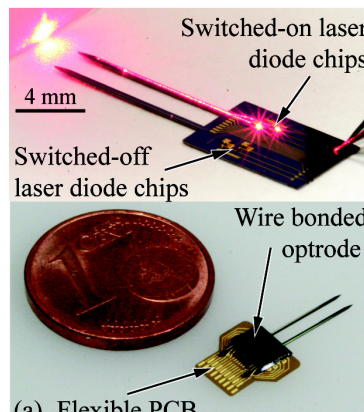

(a) Flexible PCB

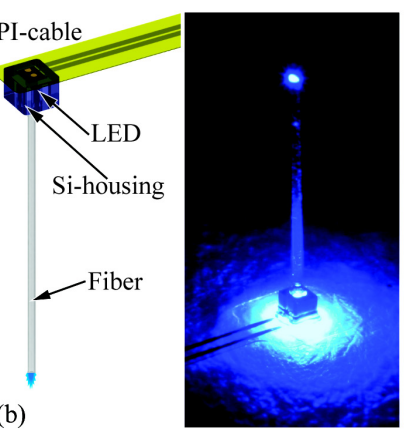

(b)
Fig. 2: Optrodes of (a) planar design with laser diodes and waveguides on slender Si probe shafts [4] and (b) miniaturized light source using LED chip mounted in Si housing and interfaced by polyimide (PI) cable (adapted from [18].

channel proteins, so-called opsins, variants of which can be expressed selectively in different types of neuronal cells. Optogenetic experiments require light of appropriate optical wavelengths to be dissipated in the neuronal network under study.

Traditionally this has been done using optical fibers connected to light sources external to the subject. In the absence of suitable optical couplers with rotational freedom, the relatively stiff connections severely restrict the freedom of motion of animals and thus are disturbing studies with freely behaving animals.

One idea to overcome this limitation has been to integrate the light sources directly onto the probes. Thereby the fiber connection is replaced by a purely electrical connection of the probe either to a telemetric headstage or through highly flexible PI cables. Figure 2 shows two examples of such systems [4][18]. In the first \{Fig. 2(a)\}, four laser diodes are integrated on the base of a twoshank probe, the light being guided to the vicinity of recording sites near the probe tip by SU- 8 waveguides. In the second case \{Fig. 2(b)\}, a light emitting diode (LED) is mounted on a silicon platform carrying a short optical fiber.

\section{Three-dimensional probes}

In order to arrange stimulation and recording sites into even more powerful three-dimensional (3D) arrays we have explored successively refined assembly techniques. Probes of the planar type described above were inserted into dedicated platforms with micromachined bays for hosting probe bases. In a first generation, the challenge of the vertical-to-horizontal lead transfer from probe to base was addressed by electroplated $\mathrm{Au}$ contacts overhanging the bays [19]. Relaxing the severe geometrical tolerances of this approach, the bays were later enhanced by thermomechanical actuators pressing the bases against the contacts on a platform/cable assembly [20]. A third design relied on bridging the gaps between the contacts on the platform and the basis by electroplating [21].

Most recently, we have explored a modular system shown in Fig. 3 with spacer modules separating probe levels with a high angular accuracy better than $1^{\circ}$, both in the plane of the probe and in the out-of-plane direction [22].

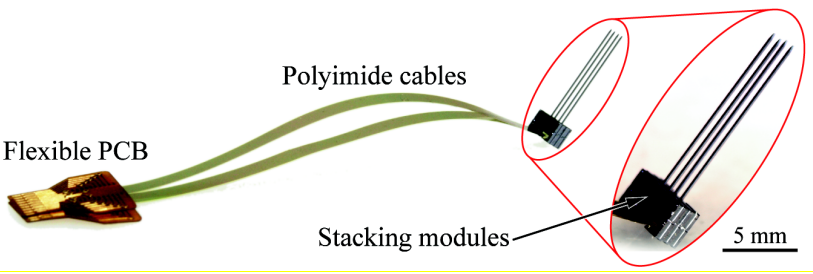

Fig. 3: 3D probe array based on stacking modules for probe alignment (adapted from [22]).
Fig. 1: Selected intracerebral neural probes: (a) passive probes with up to 64 channels (ch) and (b) active EDC probes with on-shaft circuitry for selecting eight out of 188 channels per shaft.

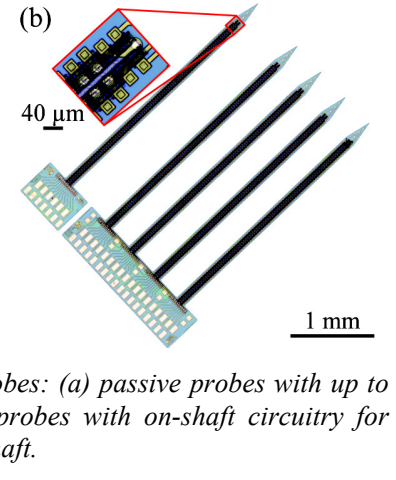

3 


\section{Probe assemblies and handling tools}

In view of the widely varying methods and skills of experimental neuroscientists, a lesson well learnt is that there is no onefits-all solution for handling the probes and interconnecting them to the experimentalist's preferred instrumentations. For this reason, customized interconnection solutions based on printed circuit boards for direct probe mounting or indirect connection via flexible PI cables with Pt lines have been custom-designed. We have realized assemblies compatible with vacuum chucks, micromanipulators, microdrives, and autonomous headstages, among others.

\section{CONCLUSION AND OUTLOOK}

BRAINLINKS-BRAINTOOLS is currently taking up speed through the projects outlined above. In the meantime a range of mature complementary neurotechnological projects independent of this funding source is being pursued at IMTEK, including the ECfunded projects NeuroSeeker, LifeHand2, and BrainCon. Spin-off activities are deployed by $\mathrm{CorTec}$ for the commercialization of ECoGs for human applications, and Atlas Neuroengineering for passive and active probes and assemblies. Overall these activities have created in Freiburg a hub of competence in neurotechnology embedded in a dense network of user groups.

In the early phase, most of the MEMS tools described in this paper are destined for acute and sub-chronic applications with animal models. Their transfer to human subjects will require a prolonged effort in order to ensure their minimal invasiveness and long-term reliability.

\section{ACKNOWLEDGMENTS}

Funding by the DFG through project no. EXC1086 BRAINLINKS-BRAINTOOLS is gratefully acknowledged.

\section{REFERENCES}

[1] www.brainlinks-braintools.uni-freiburg.de.

[2] K. Seidl, M. Schwaerzle, I. Ulbert, H.P. Neves, O. Paul, P. Ruther, "CMOS-Based High-Density Silicon Microprobe Arrays for Electronic Depth Control in Intracortical Neural Recording - Characterization and Application", J. Microelectromech. Syst., 21, 1426-1435 (2012).

[3] J. Brunne, U. Wallrabe, "Tunable MEMS Axicon Mirror Arrays", Optics Letters, 38 (11), 1939-1941 (2013).

[4] M. Schwaerzle, K. Seidl, U. Schwarz, O. Paul, P. Ruther, "Ultracompact Optrode With Integrated Laser Diode Chips and SU-8 Waveguides for Optogenetic Applications", Proc. IEEE MEMS Conf. 2013, Taipei, 2013, pp. 1029-1032.

[5] C. Henle, M. Schuettler, J. Rickert, T. Stieglitz, "Towards Electrocorticographic Electrodes for Chronic Use in BCI Applications", in: A. Brendan et al. (eds.) Towards practical $B C I s$, Springer, (2012) pp. 75-96.

[6] O.G. Gruschke, R. Kamberger, J. Hoefflin, J.G. Korvink, "Water-soluble sacrificial layer enables ultra low-cost integration of magnetic resonance microcoils with $100 \%$ filling factor", Dig. Tech. Papers Transducers 2013, Barcelona, Spain, 2013, pp. 132-134.

[7] J.-B. Hövener, N. Schwaderlapp, T. Lickert, S.B. Duckett, R.E. Mewis, L.A.R. Highton, S.M. Kenny, G.G.R. Green, D. Leibfritz, J.G. Korvink, J. Hennig, D. von Elverfeldt, "A hyperpolarized equilibrium for magnetic resonance", Nature Comm., 4, 2946 (2013).

[8] M.C. Wapler, J. Leupold, J. Dragonu, D. von Elverfeldt, M. Zaitsev, U. Wallrabe, "Magnetic Properties of Materials for
MR Engineering, Micro-MR and Beyond", J. Magnetic Resonance (2014), in press.

[9] R. Rostek, J. Kottmeier, S. Urban, M. Kratschmer, J. Jaklin, M. Cichosz, V. Sklyarenko, P. Woias, "Thermoelectric Properties of Electrodeposited and Annealed $\mathrm{Bi}_{2} \mathrm{Te}_{3}$ and $\mathrm{Sb}_{2} \mathrm{Te}_{3}$ ", Proc. 32nd Intl. Conf. on Thermoelectrics (ICT2013), Kobe, Japan.

[10] K. Wise, A. Sodagar, Y. Yao, M. Gulari, G. Perlin, K. Najafi, "Microelectrodes, Microelectronics, and Implantable Neural Microsystems", Proc. IEEE, 96, 1184-1202 (2008).

[11] P. Campbell, K. Jones, R. Huber, K. Horch, R. Normann, “A Silicon-Based, Three-Dimensional Neural Interface: Manufacturing Processes for an Intracortical Electrode Array", IEEE Trans. Biomed. Eng., 38, 758-768 (1991).

[12] S. Herwik, O. Paul, P. Ruther, "Ultrathin Silicon Chips of Arbitrary Shape by Etching Before Grinding", J. Microelectromech. Syst., 20, 791-793 (2011).

[13] S. Herwik, S. Kisban, A.A.A. Aarts, K. Seidl, G. Girardeau, K. Benchenane, M.B. Zugaro, S.I. Wiener, O. Paul, H.P. Neves, P. Ruther, "Fabrication Technology for Silicon-based Microprobe Arrays Used in Acute and Sub-chronic Neural Recording", J. Micromech. Microeng., 19, 074008 (2009).

[14] S. Spieth, O. Brett, K. Seidl, A.A.A. Aarts, M.A. Erismis, S Herwik, F. Trenkle, S. Tätzner, J. Auber, M. Daub, H.P. Neves, R. Puers, O. Paul, P. Ruther, R. Zengerle, “A Floating 3D Silicon Microprobe Array for Neural Drug Delivery Compatible with Electrical Recording”, J. Micromech. Microeng., 21, 125001 (2011).

[15] O. Frey, T. Holtzman, R. McNamara, D. Theobald, P. van der Wal, N. de Rooij, J. Dalley, M. Koudelka-Hep, "Enzymebased Choline and l-glutamate Biosensor Electrodes on Silicon Microprobe Arrays", Biosensors Bioelectronics, 26, 477484 (2010)

[16] B. Dombovári, R. Fiáth, B.P. Kerekes, E. Tóth, L. Wittner, D. Horváth, K. Seidl, S. Herwik, T. Torfs, O. Paul, P. Ruther, H.P. Neves, I. Ulbert, "In Vivo Validation of the Electronic Depth Control Probes", Biomed. Tech, 11, 1-7 (2013).

[17] F. Zhang, A.M. Aravanis, A. Adamantidis, L. Lecea, K. Deisseroth, "Circuit-breakers: Optical Technologies for Probing Neural Signals and Systems", Nat. Rev. Neurosci., 8, 577581, (2007).

[18] M Schwaerzle, P. Elmlinger, O. Paul, P. Ruther, "Miniaturized Tool for Optogenetics Based on an LED and an Optical Fiber Interfaced by a Silicon Housing”, IEEE EMBC, 2014 Chicago, submitted.

[19] A.A.A. Aarts, H.P. Neves, R.P. Puers, C.V. Hoof, "An Interconnect for Out-of-plane Assembled Biomedical Probe Arrays", J. Micromech. Microeng., 18, 064004 (2008).

[20] S. Herwik, T. Holzhammer, O. Paul, P. Ruther, "Out-of-plane Assembly of 3D Neural Probe Arrays Using a Platform With SU-8-based Thermal Actuators", Dig. Tech. Papers Transducers Conf. 2011, Beijing, China (2011), pp. 2323-2326.

[21] F. Barz, T. Holzhammer, O. Paul, P. Ruther, "Novel Technology for the In-plane to Out-of-plane Transfer of Multiple Interconnection Lines in 3D Neural Probes", Dig. Tech. Papers Transducers 2013, Barcelona, Spain (2013), pp. 884-887.

[22] F. Barz, O. Paul, P. Ruther, "3D-Assemblierungsverfahren für intrakortikale Sondenarrays", Proc. Mikrosystemtechnik Kongress, Aachen, Germany (2013), pp. 131-134.

\section{CONTACT}

*O. Paul, phone: +49-761-2037191; paul@imtek.uni-freiburg.de 